\title{
Existence Results for a Nonlocal Problem Involving the $p$-Laplace Operator
}

\author{
Mustafa Avci \\ Faculty of Economics and Administrative Sciences, Batman University, 72000-Batman, Turkey \\ *Corresponding Author: avcixmustafa@gmail.com
}

Copyright (C2014 Horizon Research Publishing All rights reserved.

\begin{abstract}
The present paper deals with a nonlocal problem under homogeneous Dirichlet boundary conditions, set in a bounded smooth domain $\Omega$ of $\mathbb{R}^{N}$. The problem studied is a stationary version of the original Kirchhoff equation involving the $p$-Laplace operator. The question of the existence of weak solutions is treated. Using variational approach and applying the Mountain Pass Theorem together with Fountain theorem, the existence and multiplicity of solutions is obtained in the Sobolev space $W^{1, p}(\Omega)$.
\end{abstract}

Keywords Variational Method, Nonlocal Problem, Mountain-pass Theorem, Fountain Theorem

MSC: 35D05; 35J60; 35J70; 58E05

\section{Introduction}

We study the existence and multiplicity of solutions for a nonlocal elliptic equation with Dirichlet zero-boundary condition, i.e., $p$-Kirchhoff equation of the following type:

$$
\begin{gathered}
-M\left(\int_{\Omega} \frac{1}{p}\left(|\nabla u|^{p}+|u|^{p}\right) d x\right)\left(\Delta_{p} u-|u|^{p-2} u\right)=f(x, u), \text { in } \Omega, \\
u=0, \text { on } \partial \Omega,
\end{gathered}
$$

where $\Omega \subset \mathbb{R}^{N}(N \geq 3)$ is a bounded domain with smooth boundary $\partial \Omega, M$ is a continuous function, $f$ satisfies Carathéodory condition and $1<p<N$.

In problem $(\mathbf{P})$, if we consider the case $p=2$, and replace the nonlocal term $M\left(\int_{\Omega} \frac{1}{2}\left(|\nabla u|^{2}+|u|^{2}\right) d x\right)$ by $M\left(\int_{\Omega}|\nabla u|^{2} d x\right)$, then we get the equation

$$
-M\left(\int_{\Omega}|\nabla u|^{2} d x\right) \Delta u=f(x, u) \text { in } \Omega
$$

which is related to the stationary analog of the Kirchhoff equation:

$$
u_{t t}-M\left(\int_{\Omega}\left|\nabla_{x} u\right|^{2} d x\right) \Delta_{x} u=f(x, t)
$$

where $M(s)=a s+b, a, b>0$. It was proposed by Kirchhoff in [10] as an extension of the classical D'Alambert's wave equations for free vibrations of elastic strings. The Kirchhoff model takes into account the length changes of the spring produced by transverse vibrations. Equation (1.2) received much attention and an abstract framework to the problem was proposed after the work [11]. Some interesting and further results can be found in [4, 14] and the references there in. In addition, (1.1) has important physical and biological background. There are many authors who pay more attention to this equation. In particularly, authors concerned with the existence of solutions for (1.1) with zero Dirichlet boundary condition via Galerkin method and built the variational frame in [2, 13]. More recently, Perera and Zhang obtained solutions of a class of nonlocal quasilinear elliptic boundary value problems using variational methods, invariant sets of descent flow, Yang index and critical groups [15, 21].

If the nonlocal term $M\left(\int_{\Omega} \frac{1}{2}\left(|\nabla u|^{2}+|u|^{2}\right) d x\right)$ replaced by $M\left(\int_{\Omega}|u|^{2} d x\right)$, then the equation

$$
-M\left(\int_{\Omega}|u|^{2} d x\right) \Delta u=f(x, u), \text { in } \Omega
$$


arises in numerous physical models such as systems of particles in thermodynamical equilibrium via gravitational (Coulomb) potential, 2-D fully turbulent behavior of real flow, thermal tunaway in Ohmic Heating, shear bands in metal deformed under high starin rates,among others. Because of importance, in [7, 17], the authors similarly studied the existence of solutions for (1.3) with zero Dirichlet boundary condition.

On the other hand, elliptic equations with nonlinear boundary conditions have become rather an active area of research; see $[3,5,6,16,19,22]$ and references therein. Those references present necessary and sufficient conditions of solutions of elliptic equations with nonlinear boundary conditions.

In the present paper, we deal with the existence of solutions for an elliptic equation $(\mathbf{P})$ with zero Dirichlet boundary condition based on variational method. The problem $(\mathbf{P})$ involves the $p$-Laplacian or the $p$-Laplace operator $\Delta_{p} u:=\operatorname{div}\left(|\nabla u|^{p-2} \nabla u\right)$ with $p>1$. The case of $p=2$, i.e., linear case of $(\mathbf{P})$, has been investigated in [19], while the general case of $p>1$ will be studied in the present paper. When $p \neq 2$, the $p$-Laplace operator $\Delta_{p}$ is nonlinear. Note also that for $p \neq 2$, the $p$-Laplace operator is $(p-1)$-homogeneous but not additive. For this reason some of the authors, in particular those who work in ODEs, call equations involving the $p$-Laplacian "half-linear" equations. The word "half-linear" reflects the fact that "one half" of the properties of linearity (i.e. additivity) is lost, while "one half" of the properties is preserved (i.e. homogeneity). The $p$-Laplace operator is very popular in nonlinear analysis and appears particularly in describing the behavior of compressible fluid in a homogeneous isotropic rigid porous medium (see e.g. [8, 12, 18]).

\section{Preliminaries}

Let $p \in \mathbb{R}$ with $1<p<\infty$; we set

$$
L^{p}(\Omega)=\left\{u: \Omega \rightarrow \mathbb{R} ; u \text { is measurable and }|u|^{p} \in L^{1}(\Omega)\right\}
$$

with

$$
|u|_{p}=\left(\int_{\Omega}|u|^{p} d x\right)^{1 / p}
$$

It is well-known that $L^{p}(\Omega)$ is a Banach space for any $p, 1 \leq p \leq \infty$.

Let $W^{1, p}(\Omega)$ be the usual Sobolev space, i.e., $W^{1, p}(\Omega)=\left\{u \in L^{p}(\Omega): \nabla u \in L^{p}(\Omega)\right\}$ which is endowed with norm

$$
\|u\|=\left(\int_{\Omega}\left(|\nabla u|^{p}+|u|^{p}\right) d x\right)^{1 / p} .
$$

Then $W^{1, p}(\Omega)$ is a Banach space. Moreover, for $p \in(1, \infty)$, it is reflexive.

Denition 1 We say that $u \in W^{1, p}(\Omega)$ is a weak solution of $(\mathbf{P})$ if

$$
M\left(\int_{\Omega} \frac{1}{p}\left(|\nabla u|^{p}+|u|^{p}\right) d x\right)\left(\int_{\Omega}\left(|\nabla u|^{p-2} \nabla u \nabla \varphi+|u|^{p-2} u \varphi\right) d x\right)=\int_{\Omega} f(x, u) \varphi d x
$$

where $\varphi \in W^{1, p}(\Omega)$. 
The energy functional corresponding to problem $(\mathbf{P})$ is defined as $I: W^{1, p}(\Omega) \rightarrow \mathbb{R}$,

$$
I(u)=\widehat{M}\left(\int_{\Omega} \frac{1}{p}\left(|\nabla u|^{p}+|u|^{p}\right) d x\right)-\int_{\Omega} F(x, u) d x,
$$

where $\widehat{M}(t)=\int_{0}^{t} M(s) d s$ and $F(x, u)=\int_{0}^{u} f(x, s) d s$. Thanks to the growth condition ( $\left.\mathbf{f}_{1}\right)$, it is not difficult to show that $I \in C^{1}\left(W^{1, p}(\Omega), \mathbb{R}\right)$, and

$$
\left\langle I^{\prime}(u), \varphi\right\rangle=M\left(\int_{\Omega} \frac{1}{p}\left(|\nabla u|^{p}+|u|^{p}\right) d x\right) \int_{\Omega}\left(|\nabla u|^{p-2} \nabla u \nabla \varphi+|u|^{p-2} u \varphi\right) d x-\int_{\Omega} f(x, u) \varphi d x,
$$

for any $u, \varphi \in W^{1, p}(\Omega)$. Hence, we can infer that critical points of functional $I$ are the weak solutions for problem $(\mathbf{P})$.

Proposition $2[1]$ Let $\Omega$ be an open and bounded subset of $\mathbb{R}^{N}$, with $N \geq 3$. Then $W^{1, p}(\Omega)$ is embedded continuously in $L^{q}(\Omega)$, denoted by $W^{1, p}(\Omega) \hookrightarrow L^{q}(\Omega)$, for every $q \in\left[1, p^{*}\right]$, where $p^{*}=N p / N-p$. The embedding is compact if and only if $q \in\left[1, p^{*}\right)$.

Proposition $3[9]$ Let $X$ be a Banach space and let define the functional $\Lambda=\int_{\Omega}\left(|\nabla u|^{p}+|u|^{p}\right) d x$. Then $\Lambda: X \rightarrow \mathbb{R}$ is convex. The mapping $\Lambda^{\prime}: X \rightarrow X^{*}$ is a strictly monotone, bounded homeomorphism, and of $\left(S_{+}\right)$ type, namely

$$
u_{n} \rightarrow u \text { in } X \text { and } \varlimsup_{n \rightarrow \infty}\left\langle\Lambda^{\prime}\left(u_{n}\right), u_{n}-u\right\rangle \leq 0 \text { implies } u_{n} \rightarrow u \text { in } X .
$$

Denition 4 Let $X$ be a Banach space and $J: X \rightarrow \mathbb{R}$ a $C^{1}$-functional. We say that a functional $J$ satisfies the Palais-Smale condition ((PS) for short), if any sequence $\left\{u_{n}\right\}$ in $X$ such that $\left\{J\left(u_{n}\right)\right\}$ is bounded and $J^{\prime}\left(u_{n}\right) \rightarrow 0$ as $n \rightarrow \infty$, admits a convergent subsequence.

\section{Main results and proofs}

In what follows, the following assumptions related to problem $(\mathbf{P})$ are considered as far as the converse is explicitely stated.

$\left(\mathbf{M}_{1}\right) M: \mathbb{R}^{+} \rightarrow \mathbb{R}^{+}$is a continuous function and satisfies the growth condition

$$
A t^{\alpha-1} \leq M(t) \leq B t^{\alpha-1}
$$

for all $t>0$, where $A, B$ and $\alpha$ are positive constants such that $\frac{B}{A}>\alpha>1$;

$\left(\mathbf{f}_{1}\right) f: \bar{\Omega} \times \mathbb{R} \rightarrow \mathbb{R}$ satisfies Carathéodory condition and the subcritical growth conditions

$$
|f(x, t)| \leq C|t|^{q-1},
$$

for all $x \in \bar{\Omega}$ and for all $t \in \mathbb{R}$, where $C$ is a positive constant and $1<p<q<p^{*}=(N p) /(N-p)$;

$\left(\mathbf{f}_{2}\right) f(x, t)=o\left(|t|^{\alpha p-1}\right), t \rightarrow 0$ for $x \in \bar{\Omega}$ uniformly, where $q>\alpha p^{\alpha}$;

$\left(\mathbf{f}_{3}\right) f(x,-t)=-f(x, t), x \in \bar{\Omega}, t \in \mathbb{R}$;

(AR) Ambrosetti-Rabinovitz's type condition holds, i.e.,

$\exists K>0, \theta>\frac{B}{A} p$ such that

$$
0 \leq \theta F(x, t) \leq f(x, t) t,|t| \geq K \text { a.e. } x \in \bar{\Omega} .
$$

The first main result of the present paper is:

Theorem 5 Suppose $\left(\mathbf{M}_{1}\right),(\mathbf{A R}),\left(\mathbf{f}_{1}\right)$ and $\left(\mathbf{f}_{2}\right)$ hold. Then $(\mathbf{P})$ has at least one nontrivial weak solution. To obtain the results of Theorem 5 , we need the following two lemmas.

Lemma 6 Suppose $\left(\mathbf{M}_{1}\right),(\mathbf{A R})$ and $\left(\mathbf{f}_{1}\right)$ hold. Then I satisfies the $(\mathbf{P S})$ condition.

Let assume that there exists a sequence $\left\{u_{n}\right\}$ in $W^{1, p}(\Omega)$ such that

$$
\left|I\left(u_{n}\right)\right| \leq c \text { and } I^{\prime}\left(u_{n}\right) \rightarrow 0 .
$$

$$
\begin{aligned}
c+\left\|u_{n}\right\| & \geq I\left(u_{n}\right)-\frac{1}{\theta}\left\langle I^{\prime}\left(u_{n}\right), u_{n}\right\rangle \\
& =\widehat{M}\left(\frac{\left\|u_{n}\right\|^{p}}{p}\right)-\int_{\Omega} F\left(x, u_{n}\right) d x-\frac{1}{\theta}\left[M\left(\frac{\left\|u_{n}\right\|^{p}}{p}\right)\left\|u_{n}\right\|^{p}-\int_{\Omega} f\left(x, u_{n}\right) u_{n} d x\right] \\
& \geq A \int_{0}^{\frac{\left\|u_{n}\right\|^{p}}{p}} s^{\alpha-1} d s-\int_{\Omega} F\left(x, u_{n}\right) d x-\frac{1}{\theta}\left[B\left\|u_{n}\right\|^{p(\alpha-1)}\left\|u_{n}\right\|^{p}-\int_{\Omega} f\left(x, u_{n}\right) u_{n} d x\right] \\
& =\frac{A}{\alpha p^{\alpha}}\left\|u_{n}\right\|^{p \alpha}-\frac{B}{\theta \alpha p^{\alpha-1}}\left\|u_{n}\right\|^{p \alpha}+\left[\int_{\Omega}\left(\frac{1}{\theta} f\left(x, u_{n}\right) u_{n}-F\left(x, u_{n}\right)\right) d x\right] \\
& \geq\left(\frac{A}{\alpha p^{\alpha}}-\frac{B}{\theta \alpha p^{\alpha-1}}\right)\left\|u_{n}\right\|^{p \alpha}-c
\end{aligned}
$$


Dividing the above inequality by $\left\|u_{n}\right\|^{p \alpha}$ and passing to the limit as $n \rightarrow \infty$ we obtain a contradiction. It follows that $\left\{\left\|u_{n}\right\|\right\}$ is bounded in $W^{1, p}(\Omega)$. Thus, there exists $u \in W^{1, p}(\Omega)$ such that passing to a subsequence, still denoted by $\left\{u_{n}\right\}$, it converges weakly to $u \in W^{1, p}(\Omega)$.

Since $\Omega$ is bounded, we have the compact embedding $W^{1, p}(\Omega) \hookrightarrow L^{q}(\Omega)$. Therefore,

$$
\begin{aligned}
u_{n} & \rightarrow u \text { in } W^{1, p}(\Omega), \\
u_{n} & \rightarrow u \text { in } L^{q}(\Omega), \\
u_{n}(x) & \rightarrow u(x) \text { a.e. in } \Omega .
\end{aligned}
$$

By (3.1), we have $\left\langle I^{\prime}\left(u_{n}\right), u_{n}-u\right\rangle \rightarrow 0$. Thus

$$
\begin{aligned}
& \left\langle I^{\prime}\left(u_{n}\right), u_{n}-u\right\rangle \\
= & M\left(\frac{\left\|u_{n}\right\|^{p}}{p}\right)\left(\int_{\Omega}\left(\left|\nabla u_{n}\right|^{p-2} \nabla u_{n}\left(\nabla u_{n}-\nabla u\right)+\left|u_{n}\right|^{p-2} u_{n}\left(u_{n}-u\right)\right) d x\right) \\
& -\int_{\Omega} f(x, u)\left(u_{n}-u\right) d x \rightarrow 0 .
\end{aligned}
$$

From $\left(\mathbf{f}_{1}\right)$ and Hölder inequality, it follows

$$
\begin{aligned}
\left|\int_{\Omega} f\left(x, u_{n}\right)\left(u_{n}-u\right) d x\right| & \leq\left. C\left|\int_{\Omega}\right| u_{n}\right|^{q-2} u_{n}\left(u_{n}-u\right) d x|+C| \int_{\Omega}\left(u_{n}-u\right) d x \mid \\
& \leq\left.\left. C|| u_{n}\right|^{q-1}\right|_{q^{\prime}}\left|u_{n}-u\right|_{q}+C \int_{\Omega}\left|u_{n}-u\right| d x
\end{aligned}
$$

If we consider the relations given in (3.2), we get

$$
\int_{\Omega} f\left(x, u_{n}\right)\left(u_{n}-u\right) d x \rightarrow 0 .
$$

Hence,

$$
M\left(\frac{\left\|u_{n}\right\|^{p}}{p}\right)\left(\int_{\Omega}\left(\left|\nabla u_{n}\right|^{p-2} \nabla u_{n}\left(\nabla u_{n}-\nabla u\right)+\left|u_{n}\right|^{p-2} u_{n}\left(u_{n}-u\right)\right) d x\right) \rightarrow 0 .
$$

From $\left(\mathbf{M}_{1}\right)$, it follows

$$
\int_{\Omega}\left(\left|\nabla u_{n}\right|^{p-2} \nabla u_{n}\left(\nabla u_{n}-\nabla u\right)+\left|u_{n}\right|^{p-2} u_{n}\left(u_{n}-u\right)\right) d x \rightarrow 0 .
$$

Since the functional (3.3) is of type $\left(S_{+}\right)$(see Proposition 3 ), we get $u_{n} \rightarrow u$ in $W^{1, p}(\Omega)$. $I$ satisfies (PS) condition.

Lemma 7 Suppose $\left(\mathbf{M}_{1}\right),(\mathbf{A R}),\left(\mathbf{f}_{1}\right)$ and $\left(\mathbf{f}_{2}\right)$ hold. Then the following statements hold:

(i) There exist two positive real numbers $\gamma$ and a such that $I(u) \geq a>0, u \in W^{1, p}(\Omega)$ with $\|u\|=\gamma$;

(ii) There exists $u \in W^{1, p}(\Omega)$ such that $\|u\|>\gamma, I(u)<0$.

PROOF. $(i)$ Let $\|u\|<1$. Then by $\left(\mathbf{M}_{1}\right)$, we have

$$
\begin{aligned}
I(u) & =\widehat{M}\left(\frac{\left\|u_{n}\right\|^{p}}{p}\right)-\int_{\Omega} F(x, u) d x \\
& \geq \frac{A}{\alpha p^{\alpha}}\|u\|^{p \alpha}-\int_{\Omega} F(x, u) d x .
\end{aligned}
$$

By the continuous embeddings $W^{1, p}(\Omega) \hookrightarrow L^{q}(\Omega)$ and $W^{1, p}(\Omega) \hookrightarrow L^{\alpha p}(\Omega)$, there exist positive constants $C_{0}$ and $C_{1}$ such that

$$
|u|_{q} \leq C_{0}\|u\| \text { and }|u|_{\alpha p} \leq C_{1}\|u\|, \quad \forall u \in W^{1, p}(\Omega) .
$$

Let $\varepsilon>0$ be small enough such that $\varepsilon C_{1}^{\alpha p} \leq \frac{A}{2 \alpha p^{\alpha}}$. Then, using $\left(\mathbf{f}_{1}\right)$ and $\left(\mathbf{f}_{2}\right)$, we get $F(x, t) \leq \varepsilon|t|^{\alpha p}+C_{\varepsilon}|t|^{q}$, for all $x \in \bar{\Omega}$ and $t \in \mathbb{R}$. Therefore, by $\left(\mathbf{M}_{1}\right)$ and (3.4), it follows

$$
\begin{aligned}
I(u) & \geq \frac{A}{\alpha p^{\alpha}}\|u\|^{p \alpha}-\varepsilon \int_{\Omega}|u|^{\alpha p} d x-C_{\varepsilon} \int_{\Omega}|u|^{q} d x \\
& \geq \frac{A}{\alpha p^{\alpha}}\|u\|^{p \alpha}-\varepsilon C_{1}^{\alpha p}\|u\|^{\alpha p}-C_{\varepsilon} C_{0}^{q}\|u\|^{q} \\
& \geq\left(\frac{A}{\alpha p^{\alpha}}-\varepsilon C_{1}^{\alpha p}\right)\|u\|^{\alpha p}-C_{\varepsilon} C_{0}^{q}\|u\|^{q} .
\end{aligned}
$$


Since $\|u\|<1$ and $q>\alpha p$, there exist two positive real numbers $\gamma$ and $a$ such that $I(u) \geq a>0, u \in W^{1, p}(\Omega)$ with $\|u\|=\gamma \in(0,1)$.

(ii) From (AR), one easily deduces

$$
F(x, t) \geq c|t|^{\theta},|t| \geq K \text { a.e. } x \in \bar{\Omega} .
$$

Moreover, when $t>1$ large enough, from $\left(\mathbf{M}_{1}\right)$, we obtain that

$$
\widehat{M}(t) \leq \frac{B}{\alpha} t^{\alpha} \leq \frac{B}{\alpha} t^{p \alpha}
$$

Hence, for $\omega \in W^{1, p}(\Omega) \backslash\{0\}$ and $t>1$ we have

$$
\begin{aligned}
I(t \omega) & =\widehat{M}\left(\frac{\|t \omega\|^{p}}{p}\right)-\int_{\Omega} F(x, t \omega) d x \\
& \leq \frac{B}{\alpha p^{\alpha}} t^{p \alpha}\|\omega\|^{p \alpha}-c t^{\theta} \int_{\Omega}|\omega|^{\theta} d x-c .
\end{aligned}
$$

Since $\theta>\alpha p$, we conclude $I(t \omega) \rightarrow-\infty$ as $t \rightarrow+\infty$.

PROOF. Proof of Theorem 5 From Lemma 6, Lemma 7 and the fact that $I(0)=0, I$ satisfies the Mountain Pass theorem (see [20]). Therefore, $I$ has at least one nontrivial critical point, i.e., (P) has a nontrivial weak solution. We are done.

The second main result of the present paper is:

Theorem 8 Suppose $\left(\mathbf{M}_{1}\right),(\mathbf{A R}),\left(\mathbf{f}_{1}\right)$ and $\left(\mathbf{f}_{3}\right)$ hold, then I has a sequence of critical points $\left\{u_{n}\right\}$ such that $I\left(u_{n}\right) \rightarrow+\infty$ and $(\mathbf{P})$ has infinite many pairs of solutions.

Let $X$ be a reflexive and separable Banach space. Then there exist $\left\{e_{j}\right\} \subseteq X$ and $\left\{e_{j}^{*}\right\} \subseteq X^{*}$ such that $X=\overline{\operatorname{Span}\left\{e_{j} \mid j=1,2, \ldots\right\}}, X^{*}=\overline{\operatorname{Span}\left\{e_{j}^{*} \mid j=1,2, \ldots\right\}}$ and

$$
\left\langle e_{i}^{*}, e_{j}\right\rangle=\left\{\begin{array}{l}
1, i=j \\
0, i \neq j
\end{array}\right.
$$

For convenience, we write $X_{j}=\operatorname{Span}\left\{e_{j}\right\}, Y_{k}=\oplus_{j=1}^{k} X_{j}, Z_{k}=\oplus_{j=k}^{\infty} X_{j}$.

\section{Lemma 9 Denote}

$$
\beta_{k}:=\sup \left\{|u|_{q} \mid\|u\|=1, u \in Z_{k}\right\} \text {. }
$$

If $q<p^{*}$, then $\lim _{k \rightarrow \infty} \beta_{k}=0$.

PROOF. It is clear that, $0<\beta_{k+1} \leq \beta_{k}$ and hence $\beta_{k} \rightarrow \beta \geq 0$. Let $u_{k} \in Z_{k}$ such that $\left\|u_{k}\right\|=1$ and $0 \leq \beta_{k}-\left|u_{k}\right|_{q}<\frac{1}{k}$. Then one can find a subsequence of $\left\{u_{k}\right\}$ (still denoted by $u_{k}$ ) which converges weakly to $u$ and $\left\langle e_{j}^{*}, u\right\rangle=\lim _{k \rightarrow \infty}\left\langle e_{j}^{*}, u_{k}\right\rangle=0, j=1,2, \ldots$, which means that $u=0$ and so $u_{k} \rightarrow 0$ in $W^{1, p}(\Omega)$. By the compact imbedding $W^{1, p}(\Omega) \hookrightarrow L^{q}(\Omega)$, we have $u_{k} \rightarrow 0$ in $L^{q}(\Omega)$, and, consequently, $\lim _{k \rightarrow \infty} \beta_{k}=0$.

PROOF. Proof of Theorem 8 By Lemma 6,I satisfies (PS) condition, and from $\left(\mathbf{f}_{3}\right)$ it is also an even functional. In the sequel, we will show that if $k$ is large enough, then there exist $\rho_{k}>r_{k}>0$ such that

(i) $b_{k}:=\inf _{u \in Z_{k},\|u\|=r_{k}} I(u) \rightarrow+\infty$, as $k \rightarrow+\infty$,

(ii) $a_{k}:=\max _{u \in Y_{k},\|u\|=\rho_{k}} I(u) \leq 0$.

Therefore, to obtain the results of Theorem 8, it is enough to apply Fountain theorem (see [20]).

(i) For any $u \in Z_{k}$ with $\|u\|$ big enough, we have

$$
\begin{aligned}
I(u) & =\widehat{M}\left(\frac{\|u\|^{p}}{p}\right)-\int_{\Omega} F(x, u) d x \\
& \geq \frac{A}{\alpha p^{\alpha}}\|u\|^{p \alpha}-c \int_{\Omega}|u|^{q} d x-c_{1} \\
& \geq \frac{A}{\alpha p^{\alpha}}\|u\|^{p \alpha}-c|u|_{q}^{q} \\
& \geq \frac{A}{\alpha p^{\alpha}}\|u\|^{p \alpha}-c\|u\|^{q}
\end{aligned}
$$

Set $\|u\|=r_{k}:=\left(c q \beta_{k}^{q}\right)^{\frac{1}{p-q}}$. Then, we have

$$
\begin{aligned}
I(u) & \geq \frac{A}{\alpha p^{\alpha}}\left(c q \beta_{k}^{q}\right)^{\frac{p \alpha}{p-q}}-c\left(c q \beta_{k}^{q}\right)^{\frac{q}{p-q}}-c_{2} \\
& \geq A\left(\frac{1}{\alpha p^{\alpha}}-\frac{1}{q}\right)\left(c q \beta_{k}^{q}\right)^{\frac{p \alpha}{p-q}}-c_{2} \rightarrow \infty \text { as } k \rightarrow+\infty
\end{aligned}
$$


because $q>\alpha p^{\alpha}$ and $\beta_{k} \rightarrow 0$.

(ii) From (AR), we deduce that $F(x, t) \geq|t|^{\theta}-c$. Thus, for any $\omega \in Y_{k}$ with $\|\omega\|=1$ and $1<t=\rho_{k}$, we have

$$
\begin{aligned}
I(t \omega) & =\widehat{M}\left(\frac{\|t \omega\|^{p}}{p}\right)-\int_{\Omega} F(x, t \omega) d x \\
& \leq \frac{B}{\alpha p^{\alpha}} t^{p \alpha}\|\omega\|^{p \alpha}-c t^{\theta} \int_{\Omega}|\omega|^{\theta} d x-c \\
& \leq \frac{B}{\alpha p^{\alpha}} \rho_{k}^{p \alpha}\|\omega\|^{p \alpha}-c \rho_{k}^{\theta} \int_{\Omega}|\omega|^{\theta} d x-c
\end{aligned}
$$

Since we have $\theta>\alpha p$ and $\operatorname{dim} Y_{k}<\infty$, we obtain that $I(u) \rightarrow-\infty$ as $\|u\| \rightarrow \infty$ for $u \in Y_{k}$. The proof of Theorem 8 is completed.

\section{REFERENCES}

[1] R.A. Adams and J.J.F. Fournier, Sobolev Spaces, Academic Press, Amsterdam, The Netherlands, 2003.

[2] C.O. Alves, F.J.S.A. Corrêa and T.F. Ma: Positive solutions for a quasilinear elliptic equation of Kirchhoff type, Computers \& Math. Appl. 49 (1) (2005), 85-93.

[3] P. Amster, M.C. Mariani and O. Mendez, Nonlinear boundary conditions for elliptic equations, Electronic Journal of Dif. Equa. 144 (2008), 1-8.

[4] A. Arosio and S. Panizzi, On the well-posedness of the Kirchhoff string, Transactions of the American Math. Soc. 348 (1) (1996), 305-330.

[5] J.F. Bonder and J.D. Rossi, Existence results for the $p$-Laplacian with nonlinear boundaryconditions, J. Math. Anal. Appl. 263 (1) (2001), 195-223.

[6] K. Chaïb, Necessary and sufficient conditions of existence for a system involving the $p$-Laplacian $1<p<N$, J. Dif. Equa.189 (2) (2003), 513-525.

[7] F.J.S.A. Corrêa and D.C. De Morais Filho, On a class of nonlocal elliptic problems via Galerkin method, J. Math. Anal. Appl. 310 (1) (2005), 177-187.

[8] P. Drabek and S. Pohozaev, Positive solutions for the $p$-Laplacian: application of the fibering method, Proc. Roy. Soc. Edinburgh Sect. A, 127 (1997), 703-726.

[9] X.L. Fan and Q.H. Zhang, Existence of solutions for $p(x)$-Laplacian Dirichlet problems, Nonlinear Anal. 52 (2003), 1843-1852.

[10] G. Kirchhoff, Mechanik, Teubner, Leipzig, 1883.

[11] J. L. Lions, On some equations in boundary value problems of mathematical physics, in: Contemporary Developments in Continuum Mechanics and Partial Differential Equations (Proc. Internat. Sympos., Inst. Mat. Univ. Fed. Rio de Janeiro, Rio de Janeiro, 1977), in: North-Holland Math. Stud. 30 (1978), 284-346.

[12] P.Lundqvist, On the equation $\operatorname{div}\left(|\nabla u|^{p-2} \nabla u\right)+\lambda|u|^{p-2} u=0$, Proc. Amer. Math. Soc.109 (1990), 157-164.

[13] T.F. Ma, Remarks on an elliptic equation of Kirchhoff type, Nonlinear Analysis: Theory, Methods \& Appl. vol. 63 , no. 5-7 (2005), 967-977.

[14] K. Ono, On global solutions and blow-up solutions of nonlinear Kirchhoff strings with nonlinear dissipation, J. Math. Anal. Appl. 216 (1) (1997), 321-342.

[15] K. Perera and Z.Zhang, Nontrivial solutions of Kirchhoff-type problems via the Yang index, J. Dif. Equa. 221 (1) (2006), 246-255.

[16] S.Z. Song and C.L. Tang, Resonance problems for the $p$-Laplacian with a nonlinear boundary condition, Nonlinear Analysis: Theory, Methods \& Appl. 64 (9) (2007), 2007-2021.

[17] R. Stańczy, Nonlocal elliptic equations, Nonlinear Analysis: Theory, Methods \& Appl. 47 (5) (2001), 35793584 .

[18] P. Takác, On the number and structure of solutions for a Fredholm alternative with the $p$-Laplacian, J. Dif. Equa. 185 (2002), 306-347. 
[19] F. Wang and Y. An, Existence of Nontrivial Solution for a Nonlocal Elliptic Equation with Nonlinear Boundary Condition, Boundary Value Problems Vol. 2009, Article ID 540360, 8 pages.

[20] Willem M., Minimax Theorems, Birkhauser, Basel, 1996.

[21] Z. Zhang and K. Perera, Sign changing solutions of Kirchhoff type problems via invariant sets ofdescent flow, J. Math. Anal. Appl. 317 (2) (2006), 456-463.

[22] J.H. Zhao and P.H. Zhao, Existence of infinitely many weak solutions for the $p$-Laplacian with nonlinear boundary conditions, Nonlinear Analysis: Theory, Methods \& Appl. 69 (4) (2008), 1343-1355. 